Meta

Journal des traducteurs

Translators' Journal

\title{
Enseigner la traductologie : pour qui et à quelles fins ?
}

\section{Lieven D'hulst}

Volume 39, numéro 1, mars 1994

La traduction et l'interprétation dans la Belgique multilingue

URI : https://id.erudit.org/iderudit/002562ar

DOI : https://doi.org/10.7202/002562ar

Aller au sommaire du numéro

Éditeur(s)

Les Presses de l'Université de Montréal

ISSN

0026-0452 (imprimé)

1492-1421 (numérique)

Découvrir la revue

Citer cet article

D'hulst, L. (1994). Enseigner la traductologie : pour qui et à quelles fins ? Meta,

39(1), 8-14. https://doi.org/10.7202/002562ar d'utilisation que vous pouvez consulter en ligne.

https://apropos.erudit.org/fr/usagers/politique-dutilisation/ 


\title{
ENSEIGNER LA TRADUCTOLOGIE : POUR QUI ET À QUELLES FINS?
}

\author{
LIEVEN D'HULST \\ Université d' Anvers (RUCA-HIVT), Anvers, Belgique
}

\section{PRÉALAB̈LE}

Un thème aussi vaste que la traduction et l'interprétation dans la Belgique multilingue comprend sans doute plusieurs formes de réflexion sur ces deux pratiques verbales, sans qu'il soit au départ possible de distinguer, et les théorisations, et leur rapport exact aux pratiques. Je me proposerai dès lors d'examiner de plus près cette difficulté, qui me paraît d'une part caractéristique - au moins jusqu'à un certain point - de l'enseignement de la traductologie en Belgique, mais qui relève aussi, et de manière plus fondamentale, de présupposés sur l'agencement entre enseignement et recherche.

\section{OÙ EN EST LA TRADUCTOLOGIE EN BELGIQUE ?}

À l'image de l'activité traduisante, une intense activité traductologique se développe en Belgique, sans qu'elle puisse bénéficier jusqu'à ce jour de structures qui lui paraissent appropriées. Les instituts de formation de traducteurs et interprètes réservent dans leurs programmes une place généralement modeste à l'étude d'aspects divers de la discipline, soit sous la forme de cours généraux imposés, soit sous la forme d'options choisies en alternance avec des cours de traduction, soit, et c'est de loin le cas le plus fréquent, sous la forme d'une théorisation occasionnelle engendrée par l'exercice de traduction. Dans les deux premiers cas, on fait face à des difficultés considérables : d'une part, l'étudiant sera tenté de confronter les idées nouvelles avec celles qu'il a acquises au cours de ses travaux de traduction, et risque d'être désorienté par ce qu'il ressent comme une absence de continuité; d'autre part, il éprouvera une sorte d'isolement, d'où il lui sera toujours difficile d'éviter le vieux débat théorie-pratique. C'est bien sûr dans ce dernier que se cantonne à son tour la théorisation occasionnelle.

Certes, aussi longtemps que la formation des professeurs de traductologie n'est pas étayée par des bases scientifiques, la référence exclusive aux règles imposées par le marché de la traduction entravera des formes de recherche (historique, empirique, théorique) qui ne conduisent pas en droite ligne à des applications possibles (voir Gile 1991), en même temps que par une sorte de paradoxe apparent elle donne lieu à une promotion de perspectives scientifiques plus pures. On oublie trop souvent, en effet, que le marché de la traduction est doublé d'un marché traductologique, qui possède une relative autonomie par rapport au premier. Suggérer que celui-ci dicte (ou devrait dicter) l'offre traductologique, entraîne un rééquilibrage qui consiste à doter d'une plus-value toute attitude scientifique désintéressée face aux questions d'application.

Ces tensions latentes trouvent une expression adéquate dans la position ambiguë des instituts de formation vis-à-vis des formations de lettres issues de la tradition philologique : assurant en principe un même niveau que celles-ci, ils s'en démarquent cependant, depuis leur création il y a plusieurs décennies, par une plus forte orientation vers la pratique, par un faible degré de spécialisation, et par une infrastructure scientifique rudimentaire. Les instituts ne peuvent assurer la formation de troisième cycle conduisant au doctorat, ne 
disposent pas d'un encadrement proprement scientifique, et sont donc obligés de recruter leurs professeurs titulaires dans les départements ou facultés de lettres, où ces derniers ont acquis des compétences souvent éloignées de leurs futures charges enseignantes. Ce paradoxe illustre la suspicion dont les autorités politiques comme les universités entourent les ambitions académiques des instituts de formation, - fût-ce pour des raisons différentes : le risque d'un financement (trop) onéreux aux yeux des premières? Une concurrence estimée dangereuse étant donné le grand nombre d'étudiants concernés?

Aussi la Belgique risque-t-elle d'encourir un retard par rapport à ses voisins, en ce qu'elle tend à limiter l'apport scientifique des instituts aux domaines appliqués. Par ricochet, l'orientation plus ouvertement professionnelle qu'on observe au sein des départements de lettres fait pendant aux ambitions académiques des instituts de formation, au point que de nouvelles structures de coopération de type postgraduat commencent à prendre forme au delà des clivages imposés par l'administration. Elles proposent des modes d'enseignement et de recherche (notamment à Anvers, Leuven ou Liège) qui répondent avec efficacité à l'évolution rapide de la discipline, et qui par un revers heureux pourraient enfin rendre possible la mise en cuvre d'une conception réellement interdisciplinaire de la traductologie. Reste à savoir dans quelle mesure cette nouvelle orientation, pourtant encouragée par les autorités politiques (nationales et européennes) à une époque où les ressources financières se raréfient, rencontrera l'appui durable des instances académiques.

\section{DIDACTIQUE DE LA TRADUCTION OU DE LA TRADUCTOLOGIE ?}

Au demeurant, par plus d'un côté, la traductologie offre l'image d'une discipline victime d'une crise de croissance à l'échelle internationale. De ce fait, elle souffre mal la comparaison avec les disciplines langagières traditionnelles, tant sur le plan de la formation que sur celui de la recherche, où les progrès sont inégaux selon les branches ou les sujets. Et c'est en particulier le point d'articulation de ces deux aspects essentiels qui fait apparaître l'une des grandes faiblesses de la traductologie contemporaine.

En d'autres termes, si la didactique de la traduction se porte bien, au vu du rythme élevé des publications qui se succèdent depuis plusieurs années, il semble bien, en revanche, qu'on ne se soit guère encore interrogé sur ses rapports exacts avec la ou les théories de la traduction dont elle estime dépendre, cependant que, de leur côté, celles-ci s'enseignent souvent sans référence à des considérations de type didactique ou appliqué. Dans les deux cas fait défaut une réflexion sur la nature, l'enjeu et les aspects didactiques de l'enseignement de la traductologie, comme si les questions ayant trait à cet enseignement étaient soit confondues avec celles qui ressortissent à une didactique de la traduction, soit apparaissaient comme subsidiaires par rapport à la réflexion traductologique proprement dite. Par ailleurs, faut-il croire à l'existence possible d'un consensus sur la traductologie, et sur le ou les buts que son enseignement poursuit? Convient-il ou non de réduire sa portée à un ensemble de choix didactiques, théoriques ou autres?

Bref, une réflexion sur les tenants et aboutissants des démarches enseignantes ne doit pas passer sous silence les présupposés qui sont à l'œuvre en elles. Leur mise au jour à laquelle entend contribuer cette esquisse devrait nous aider à dissiper certains malentendus tenaces.

\section{CONTRAINTES PRAGMATIQUES}

À mesure que la traductologie accède au rang d'une discipline académique respectable, elle est adoptée par les programmes d'enseignement et de recherche, phénomène qui conduit en retour à la mise en relief active et consciente de la nouvelle autonomie acquise. Elle s'oblige corrélativement à sacrifier l'idéal d'un savoir et d'une pratique 
homogènes, unifiés autour de concepts et de méthodes partagés par ses usagers. Le lieu de la lutte pour l'identité respectée se déplace des frontières entre le champ traductif et les disciplines adjacentes vers une pluralité de champs traductologiques présentés comme tels au sein même d'un concept toujours plus abstrait à mesure qu'il doit désigner des ensembles hétérogènes. Par un effet peut-être naturel de la nouvelle situation, le débat procède par empiétement sur les voisins du champ central qu'est la théorie : mais si l'on passe ainsi de la théorie à l'analyse (expérimentale) ou l'histoire et vice versa, la théorie inspire sans retour l'enseignement de la traduction.

Qu'il s'agisse d'une théorie de la traduction humaine ou automatique, ou d'une méthode d'enseignement de la traduction, leur présentation repose dès lors sur des prémisses le plus souvent informulées, mais normalement conçues, à l'intention du récepteur, en réponse aux nécessités du principe de la pertinence, lequel repose sur une double présomption:

$[\ldots]$ the set of assumptions which the communicator intends to convey will be adequately relevant to the audience, and the stimulus produced is such that it obviates gratuitous processing effort on the audience's part. (Gutt 1990: 139)

Des contraintes pragmatiques incitent donc l'émetteur à choisir une formulation spontanément perçue comme pertinente par le récepteur, voire à mettre en œuvre un dispositif discursif propre à susciter l'adhésion maximale de ce dernier. Corrélativement, le choix et le traitement des théories traductives en contexte didactique dépendent en premier lieu de leur adéquation (implicite) à ce contexte.

\section{QUELLES THÉORIES POUR QUI?}

À prendre les choses d'un peu haut (nous verrons ci-après des modalités transitoires), on dira que le bénéficiaire des théories est soit l'étudiant de traduction (d'un niveau avancé), soit l'étudiant de traduction ou de lettres (langue ou littérature) ayant un goût pour la recherche, et, le cas échéant, se destinant à une carrière scientifique en traductologie. Dans les deux cas, mais à une échelle différente, s'opère une mise en perspective traductologique.

Une tradition dominante consiste à voir dans la théorie un complément de la pratique : apprendre à bien traduire suppose l'acquisition d'un bagage théorique peut-être mince mais solide. Certes, le recours à la théorie dépend autant de la nature et du niveau de la formation des traducteurs, que de la manière dont la théorie elle-même a été conçue. Les débats à ce sujet sont légion. Je n'en veux pour témoins que les congrès triennaux de la FIT, qui réservent une section à la théorie où la question de la théorie pour... s'avère remarquablement constante.

La théorie s'enseigne ensuite dans le cadre d'une formation plus axée sur la recherche, analogue à une formation de lettres, souvent de type comparatiste en particulier. Dans ce cas, bien entendu, s'ajouteront aux premières, selon les angles envisagés, des théories dont l'orientation pratique n'est pas obvie. Il arrive qu'un certain nombre d'entre elles connaissent un sort double, et que des maisons d'édition qui pour des raisons commerciales élargissent au public de traducteurs ou d'étudiants de traduction les volumes qu'ils publient, mettent à profit cette possibilité, — si elles ne l'encouragent pas.

Toutefois, il n'est pas aisé de rendre compte des modalités d'échange entre les deux domaines, ni des évolutions qui déterminent leurs succès relatifs; ceux parmi les articles et ouvrages qui offrent un descriptif de préférence élémentaire mais général des concepts et tendances en cours semblent à l'heure actuelle disposer de meilleures chances d'atteindre les deux publics que les études plus pointues, soit théoriques, soit analytiques ou expérimentales. La liste suivante procurée par un professeur de traduction est éloquente à ce propos: 
- Mildred L. Larson's Meaning-based Translation: A Guide to Cross-language Equivalence, Part I (1984) presents concepts as 'form' and 'meaning', 'source' and 'target' language, 'deep' and 'surface' structure, 'literal', 'idiomatic', etc.

- Christine Durieux's article "Qu'est-ce qu' une bonne traduction?» (1988) is an excellent summary of La theorie du sens and the author's opinion of literal and idiomatic translation.

- Peter Newmark's A Textbook of Translation (1988) is a helpful guide to many aspects. His defense of literal translation is opposed to that of Mme Durieux's.

- Elsa Pucciarelli's ¿Qué es la traducción? (1970) is a very simple book but very practical and direct.

- Frank Königs's various articles on the psycholinguistic approach to translation and his theory on the 'ad-hoc' and 'residual' blocks.

Students have also read Vinay and Darbelnet's chapters on le message et les procédés de traduction, some chapters of Nida's works and several articles by Coseriu, Seleskovitch and others. (P. Hörmann 1992: 60)

D'une part, on voit que le rapport entre les deux formes théoriques est celui d'une prééminence chronologique et logique de la théorie pure, expression de la recherche en progrès, sur la théorie reformulée pour les besoins de l'enseignement. D'autre part, la structure double de certains textes théoriques rend possibles des phénomènes de retardement par rapport aux progrès marqués par les premiers. Et chez certains intermédiaires comme Christiane Nord (1991) on trouvera une présentation détaillée de la possibilité de convertir en vue de la pratique de modèles théoriques (comme ceux mis au point par la linguistique textuelle). Le phénomène de retardement $s$ 'accroît encore du fait que les théories sont dissociées de leurs contextes respectifs qui en expliquaient le caractère dynamique et interactif. Les donner comme des représentations adéquates d'un état de fait - en dissimulant l'éventuelle absence de consensus à leur endroit - oblige évidemment l'auteur de méthodes d'attendre la mise au point de travaux de type vulgarisateur, plutôt que de prendre une part effective aux discussions théoriques.

Ajoutons que les usagers - comme les concepteurs - ne sont jamais à l'abri de la confusion entre théories et méthodologies (Maurice Pergnier 1993: 9), souvent parce que la lecture des unes et des autres peut évoluer dans le temps. Voire: l'on est tenté de reprendre pour le compte de la traductologie la mise en garde faite par J. Hillis Miller quant au transfert de la théorie littéraire à la critique :

It is conceivable that true literary theory, the real right thing, may be impossible to teach or to use in practical criticism. Theory may be impossible to translate in all the senses of that word, that is, impossible to transfer to another context, for example another language. (Miller $1991: 30$ )

Et lorsque des théories franchissent les frontières linguistiques, elles subissent une domestication (ou transformation) de leurs idées, notamment au service d'applications dans un contexte didactique. En résulte comme dans le cas précité (Hörmann 1992) un usage combiné de textes traduits et non traduits, mais utilisés dans un cadre changé. Le procédé engendre force questions :

How does this translation happen? How do works of theory get a new start in a new language and within a new culture? Just how does this transfer take place? How does a work of theory cross borders, occupy a new territory, and make a new place for itself in a new language? (Miller 1991 : 37)

Ce qui vaut pour l'exposé théorique vaut également, et l'on pourrait dire à plus forte raison, pour l'histoire de la discipline. Or, souvent, les théoriciens offrent de celle-ci une vue intéressée, qui n'affecte pas le degré d'originalité ou de profondeur qu'ils s'attribuent 
eux-mêmes; ils rechercheront l'adhésion du lecteur au travers d'un habile dispositif argumentatif, et notamment métaphorique, apte à écarter des théories anciennes ou concurrentes (D'hulst 1992).

Enfin, outre la tendance qui consiste en un transfert unidirectionnel de la théorie à la méthodologie, on rencontre, et de manière progressive, la mise au point de méthodologies qui intègrent dans une certaine mesure des théories didactiques (voir notamment Hönig et Kußmaul 1982).

\title{
DES «INTRODUCTIONS»
}

Il serait évidemment illusoire de penser en retour que les textes qui enseignent la traductologie à un public élargi aux non-traducteurs (ou au moins à un public qui n'y reconnaît pas en premier lieu la vertu de l'application) ne se servent pas, au moins implicitement, de techniques didactiques mises en ouvre dans d'autres processus d'apprentissage (de la linguistique, de la théorie littéraire, etc.). Le marché s'accroît en effet pour des textes de ce genre, comme l'illustre le succès des Translation Studies de Mary SnellHornby, des Translation Studies de Susan Bassnett, ou de l'Einführung in die Übersetzungswissenschaft de Werner Koller. Une vue aussi globale que possible de la discipline y est prônée - fût-elle panoramique -, et l'on y insiste aussi sur l'unité en quelque sorte organique de la théorie et de la pratique:

This study is an attempt to bridge the gap. It is not [...] a study on literary translation; it is rather an attempt to present recently developed concepts and methods, both from translation theory and linguistics, in such a way that they could be usefully employed in the theory, practice and analysis of literary translation. (M. Snell-Hornby 1988: 1)

L'ouverture aménagée vers les traducteurs et leur pratique n'exclut pas l'insistance sur des seuils qu'on s'efforce de justifier, avec des degrés qui varient selon les traditions scientifiques, par l'état même de la discipline. Ainsi Koller (1992: 10-11):

\begin{abstract}
Diese «Einführung» setzt sich das Ziel, übersetzungsrelevante Fragestellungen, Probleme und Theorien einem breiteren Leserkreis nahezubringen (natürlich insbesondere auch den Studenten und Studentinnen an den Instituten für Ubersetzen und Dolmetschen und den $\ddot{U}$ bersetzern und Übersetzerinnen in der Berfufspraxis). [...] Ob mir eine wissenschaftlichen Ansprüchen genügende, aber zugleich leserfreundliche Darstellung gelungen ist, muß dem Urteil des Lesers überlassen bleiben. Leserfreundlich heißt für mich freilich nicht «populärwissenshaftlich"; es heißt auch nicht, daß simplifiziert wird (und simplifizieren ist etwas anderes als vereinfachen) [...]; wer sich ernsthaft mit Übersetzung auseinandersetzen will, $m u \beta$ gewillt sein, die Anstrengung auf sich zu nehmen, die jede wissenschaftich-theoretische Beschäftigung kostet.
\end{abstract}

Comme l'a du reste souligné James Holmes, le nom même de la discipline est indissociable d'une représentation identitaire variable d'un pays à l'autre, et diversement sanctionnée par leurs institutions académiques.

Par ailleurs, on relève fréquemment un dédoublement en un versant descriptif (et souvent rétrospectif) de la discipline et un versant critique (et souvent prospectif), les deux pouvant, le cas échéant, faire l'objet de parties distinctes: tel est le cas de l'ouvrage de K. van Leuven-Zwart (1992), par exemple. On y retrouve, toujours informulée, une perspective historique qui révèle son utilité, sans être toutefois conduite à son terme. Au demeurant, on peut distinguer, inéquitablement présents dans les études, certains avantages implicites de l'analyse historique de la traductologie:

- L'histoire forme pour le néophyte une excellente voie d'accès à la discipline.

- Elle donne au chercheur la flexibilité intellectuelle qui lui sera nécessaire lorsqu'il s'agit pour lui d'adapter ses idées à de nouvelles manières de penser. 
- Elle incite à une plus grande tolérance à l'endroit de manières éventuellement déviantes de penser les questions de traduction, s'opposant ainsi à une adhésion aveugle à telle ou telle théorie, en rendant possible aussi une distinction à froid entre le progrès réel et la simple reformulation, celle-ci pouvant être présentée et accueillie comme originale dans des conditions spécifiques.

- Elle est pratiquement le seul moyen de retrouver l'unité d'une discipline, en montrant les parallèles et les recoupements entre des traditions de pensée et d'activité divergentes, en rapprochant le passé et le présent.

- Elle permet régulièrement aux traducteurs de se ressourcer à des modèles passés.

Il est rarissime de trouver réunis en un seul ouvrage plus de deux ou trois de ces avantages. Plus souvent, nous l'avons dit, l'histoire sert des propos argumentatifs: elle procurera au théoricien d'aujourd'hui des prédécesseurs, ou mettra au contraire en relief sa propre originalité. Les anthologies traductologiques n'échappent pas toujours à ce péril. Rares, enfin, sont les études qui s'attachent à l'historiographie de la traductologie du $X^{\mathrm{e}}$ siècle (un exemple : Larose 1987), voire de la seconde moitié, sans déboucher sur une théorie, ou sur le choix déclaré pour telle ou telle démarche, selon tel ou tel angle. Les théoriciens se plaisent ainsi à imposer aux évolutions récentes de la discipline une écriture historique qu'il faudra sans doute corriger un jour, avec quelque sévérité, selon des principes strictement historiques.

\section{Notes}

1. Voir notamment D'hulst (1993).

2. Nous n'étudierons pas ici le rôle de la traduction dans l'apprentissage des langues (étrangères), notamment à l'université. Sur cette méthode ancienne, qui connaît, semble-t-il, un regain d'intérêt au contact d'une traductologie elle-même expansive, voir Michel Ballard (1992).

3. Cf. par exemple Toury $(1992: 60)$ :

Unfortunately, however, ideas which have long been admitted into translation theory and tested in various descriptive disciplines still need propagating in the context of our thinking about the formal training of translators.

4. Nous avons étudié de ce point de vue le rôle des métaphores en traductologie contemporaine (D'hulst $1992: 33-51$ ).

5. Il n'y a pas lieu de comparer dans cette esquisse la situation présente avec des conceptions et méthodes antérieures du processus pédagogique, ni d'examiner l'évolution des moyens techniques et autres dont ce dernier se sert (manuels, syllabus, transparents, instruments électroniques, vidéos, etc.), ni même de confronter les traditions scientifiques et académiques internationales.

6. On trouve la même préoccupation chez Kitty van Leuven-Zwart (1992:9) :

Het werk als geheel kan worden gezien als de concretisering van mijn persoonlijke visie op de vertaalwetenschap : een autonome discipline die de onlosmakelijke verbondenheid van theorie en praktijk als uitgangspunt hanteert en die een plaats inruimt voor al het onderzoek dat op de éen of andere manier met vertalen in verband staat.

7. Cf. Holmes (1988: 70) :

The problem is not that the discipline is not a Wissenschaft, but that not all Wissenschaften can properly be called sciences. Just as no one today would take issue with the term Sprachwissenschaft and Literaturwissenschaft, while more than a few would question whether linguistics has yet reached a stage of precision, formalization, and paradigm formation such that it can properly be described as a science, and while practically everyone would agree that literary studies are not, and in the foreseeable future will not be, a science in the true sense of the English word, in the same way I question whether we can with any justification use a designation for the study of translating and translations that places it in the company of mathematics, physics, and chemistry, or even biology, rather than that of sociology, history, and philosophy - or for that matter of literary studies.

Voir aussi J. Lambert (1991). 


\section{RÉFÉRENCES}

BALLARD, Michel (1992) : Le commentaire de traduction anglaise, Paris, Nathan.

BASNETT, Susan (1991) : Translation Studies, Revised Edition, London / New York, Routledge.

D’HULST, Lieven (1992) : «Sur le rôle des métaphores en traductologie contemporaine», Target, 4-1, pp. 33-51.

D'HULST, Lieven (1993) : «Nieuw onderzoeksinitiatief aan UA: vertaalwetenschap komt uit catacomben», Universiteit Antwerpen, 22, pp. 13-15.

GILE, Daniel (1991) : «Methodological Aspects of Interpretation (and Translation) Research», Target, 3-2, pp. 153-174.

GUTT, Ernst-August (1990) : «A Theoretical Account of Translation - Without a Translation Theory», Target, 2-2, pp. 135-164.

MILLER, J. Hillis (1991) : «Border Crossings: Translating Theory», EurAmerica, 21-4, pp. 27-51.

HÖNIG, Hans G. et Paul KUßMAUL (1982) : Strategie der Übersetzung: Ein Lehr-und Arbeitsbuch, Tübingen, Narr.

HÖRMANN, Patricia (1992): «Introducing Theory in a Course of Translation: a Chilean Case Study», Cay Dollerup et Anne Loddegaard (Éds.), Teaching Translation and Interpreting. Training, Talent and Experience, Amsterdam/Philadelphia, John Benjamins Publishing Company, pp. 57-61.

HOLMES, James (1988): «The Name and Nature of Translation Studies», Translated! Papers on Literary Translation and Translation Studies, Amsterdam, Rodopi, pp. 76-80.

KOLLER, Werner (1992): Einführung in die Übersetzungswissenschaft, Heidelberg/Wiesbaden, Quelle \& Meyer. 4., völlig neu erarbeitete Auflage.

LAMBERT, José (1991): «Shifts, Oppositions and Goals in Translation Studies: Towards a Genealogy of Concepts», Kitty M. van Leuven-Zwart et Ton Naaijkens (Éds.), Translation Studies: The State of the Art, Amsterdam/Atlanta, Rodopi, pp. 25-37.

LAROSE, Robert (1987): Théories contemporaines de la traduction, Québec, Presses de l'Université du Québec.

NORD, Christiane (1991): Text Analysis in Translation: Theory, Methodology, and Didactic Application of a Model for Translation-Oriented Text Analysis, Amsterdam / Atlanta, Rodopi.

PERGNIER, Maurice (1993): Les fondements sociolinguistiques de la traduction, Lille, Presses Universitaires de Lille.

SNELL-HORNBY, Mary (1988) : Translation Studies. An Integrated Approach, Amsterdam / Philadelphia, John Benjamins Publishing Company.

TOURY, Gideon (1992): «"Everything Has Its Price": an Alternative to Normative Conditioning in Translator Training», Interface, 6-2, pp. 60-72.

SPERBER, Dan et Deirdre WILSON (1986): Relevance. Communication and Cognition, Oxford, Basil Blackwell.

VAN LEUVEN-ZWART, Kitty (1992): Vertaalwetenschap: ontwikkelingen en perspectieven, Muiderberg, Dick Coutinho. 